TECHNICAL REPORT

\title{
Heavy ion collider NICA at JINR
}

To cite this article: S. Kostromin and A. Sidorin 2021 JINST 16 T03003

View the article online for updates and enhancements.

The ECS is seeking candidates to serve as the

Founding Editor-in-Chief (EIC) of ECS Sensors Plus,

a journal in the process of being launched in 2021

The goal of ECS Sensors Plus, as a one-stop shop journal for sensors, is to advance the fundamental science and understanding of sensors and detection technologies for efficient monitoring and control of industrial processes and the environment, and improving quality of life and human health.

Nomination submission begins: May 18, 2021

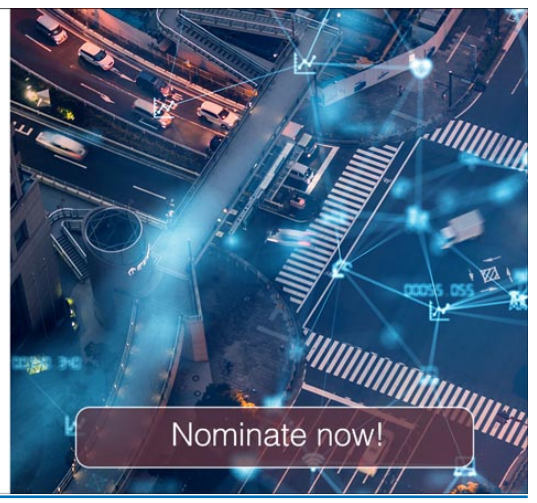




\section{Heavy ion collider NICA at JINR}

\section{S. Kostromin and A. Sidorin*}

Joint Institute for Nuclear Research, Joliet-Curie 6, Dubna 141980, Russia

Saint Petersburg State University, 13B Universitetskaya Emb., Saint Petersburg 199034, Russia

E-mail: sidorin@jinr.ru

Aвstract: Main scientific goal of the NICA/MPD project (Nuclotron-based Ion Collider fAcility/ Multi Purpose Detector) under construction at JINR (Dubna) is exploration of the phase diagram of strongly interacting matter in the region of high compression. The planned experimental program includes studies of possible signs of the phase transitions and critical phenomena in heavy ion (up to $\mathrm{Au}$ ) collisions at the center-of-mass energies up to $11 \mathrm{GeV} / \mathrm{u}$. The collider experiment provides optimum conditions for efficient energy scan measurements. Attainment of the required average luminosity of the order of $10^{27} \mathrm{~cm}^{-2} \cdot \mathrm{s}^{-1}$ for such physics experiment faces several accelerator physics and technology challenges. Contrary to high energy colliders, the luminosity of NICA case will be mostly limited by the Laslett tune shift, while the beam-beam tune shift parameter will be negligibly small. Flexible operational procedures for the beam storage and short bunch formation have been developed to provide maximum peak luminosity over wide energy range. Beam cooling is critical to counteract luminosity decay due to intra-beam scattering. NICA collider design addresses these issues and satisfies all the requirements for the colliding beams experiments.

KeYwords: Beam dynamics; Accelerator Subsystems and Technologies; Instrumentation for heavyion accelerators

\footnotetext{
${ }^{*}$ Corresponding author.
} 


\section{Contents}

1 Introduction 1

2 Structure of the facility 2

3 Challenges for heavy ion collider $\quad 4$

3.1 Luminosity of the collider 4

3.2 Space charge effects at low energy 5

$\begin{array}{lll}3.3 & \text { Regimes of the collider operation } & 7\end{array}$

4 Two concepts of the low energy collider $\quad 8$

4.1 NICA CDR 8

4.2 Present concept 9

5 Outlook 10

\section{Introduction}

NICA (Nuclotron-based Ion Collider fAcility) [1] is an international project under construction at the Joint Institute for Nuclear Research (an international intergovernmental organization in Dubna, Russia), supported by 18 member states and six associated countries. The project started in 2009 and is on track for completion in 2023. NICA, a modern accelerator facility, will support worldleading programs in long-baseline fundamental and applied research and education. The project comprises experimental studies of fundamental character in the fields of the following directions:

- relativistic nuclear physics [2];

- spin physics in the high and middle energy range of interacting particles;

- radiobiology.

Applied research based on particle beams generated at NICA is dedicated to the development of novel technologies in material science, environmental problems resolution, energy generation, particle beam therapy, and others.

An education program is one of the priority activities at JINR, as formulated in JINR Roadmap. The proposed NICA facility offers various possibilities for teaching and qualification procedures, including practice at experimental setups, diploma works, $\mathrm{PhD}$, and doctoral theses. For education of personnel, two new magister programs were actualized in 2019: control processes at megascience facilities (developed in co-operation with Saint-Petersburg State University) and Charge particle accelerators for mega-science facilities (in co-operation with Moscow Engineering Physical Institute). In co-operation with the JINR University center, the JINR educational portal was 
created [3]. It targets students and schoolchildren of the JINR Member States, young specialists, and science teachers. The portal offers courses in the MOOC format on priority JINR activities. The first courses on the NICA megaproject topics, on heavy ions and the synthesis of new elements, fundamental and applied research of nanostructures and condensed matter using neutrons have already been created and published. In addition to online courses, the portal contains links to materials visualizing JINR's basic physical facilities using 3D modeling tools, as well as to the Virtual Laboratory on Experimental Nuclear Physics.

\section{Structure of the facility}

The NICA complex (figure 1) includes:

- set of accelerators providing the particle beams for fixed target and collider experiments,

- experimental facilities,

- factory for superconducting magnet assembly and testing,

- workshops for the construction of the detector elements,

- NICA innovation center,

- required infrastructure.

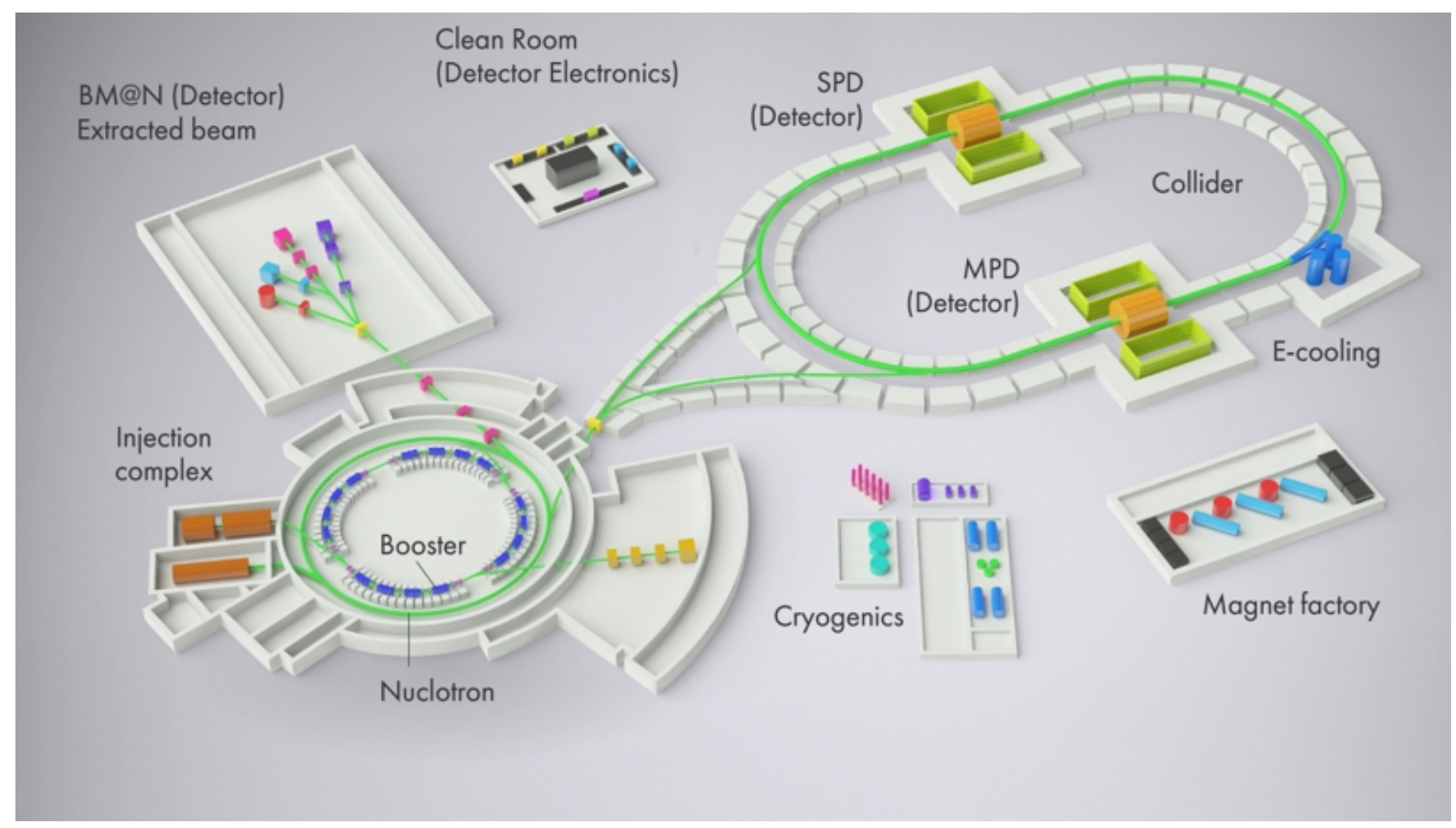

Figure 1. Sketch of the NICA complex.

The main accelerator of the NICA complex is the Nuclotron $-251.52 \mathrm{~m}$ long superconducting ion synchrotron at magnetic rigidity of about $45 \mathrm{~T} \cdot \mathrm{m}$ equipped with two injection chains: for heavy 
(including small superconducting synchrotron - the Booster) and light ions. The collider experiments will be provided at two storage rings (the possibility of slow beam acceleration is foreseen) with two interaction points at zero beam crossing angle. The Collider ring $503.04 \mathrm{~m}$ long has a racetrack shape and is based on double-aperture (top-to-bottom) superconducting magnets. The major parameters of the NICA Collider are the following: magnetic rigidity is $45 \mathrm{~T} \cdot \mathrm{m}$; maximum dipole field $1.8 \mathrm{~T}$; ion kinetic energy range from $1 \mathrm{GeV} / \mathrm{u}$ to $4.5 \mathrm{GeV} / \mathrm{u}$ for $\mathrm{Au}^{79+}$; average luminosity of $10^{27} \mathrm{~cm}^{-2} \cdot \mathrm{s}^{-1}$ for Gold ion collisions at $\sqrt{s}_{\mathrm{NN}}=9 \mathrm{GeV} / \mathrm{u}$. The maximum kinetic energy of polarized deuterons is $6 \mathrm{GeV} / \mathrm{u}$, protons $-12 \mathrm{GeV}$, the luminosity in the polarized mode is up to $10^{32} \mathrm{~cm}^{-2} \cdot \mathrm{s}^{-1}$.

The main experimental facilities are:

- Multi-Purpose Detector (MPD) aims to study hot and dense strongly interacting matter in heavy-ion (up to $\mathrm{Au}$ ) collisions at the center-of-mass energy range of max baryonic density (up to $11 \mathrm{GeV}$ ).

- Spin Physics Detector (SPD) aims to study spin physics with colliding beams of polarized deuterons and protons at the energies up to $27 \mathrm{GeV}$ (for protons).

- Baryonic Matter at Nuclotron (BM@N) - fixed target experiment at the Nuclotron extracted beams whose main goals are investigations of strange / multi-strange hyperon, hypernuclei production, and short-range correlations.

- Area for radiobiology and applied research is under development in the existing experimental building; additional experimental area for methodical investigations in hadron therapy is foreseen on the basis of the Booster extracted beams.

- The Nuclotron internal target station is equipped with six different targets: wire, strip, and film with material from hydrogen to tungsten dedicated for particle physics, spin physics, relativistic atomic physics experiments.

The facility physics program is suggested to be implemented during three stages:

Stage I

- Fixed target experiment with heavy ions

Stage II

- Basic configuration of the collider and detector

- Full configuration, heavy-ion collisions

- Collisions of heavy ions with light ions (protons)

Stage III

- Spin physics program.

Stages I and II are dedicated to the study of hot and dense strongly interacting matter in heavy ion (up to $\mathrm{Au}$ ) collisions. Peculiarities of a heavy ion collider operation at small ion kinetic energy is discussed in this article. 


\section{Challenges for heavy ion collider}

\subsection{Luminosity of the collider}

In the frame of the NICA project, the phase diagram of strongly interacting matter in the region of the high density of baryonic matter will be explored in the collider experiment with the MPD detector. The general requirement to the collider is to provide optimum operation of the detector. MPD design presumes investigation of the collisions at zero beam crossing angle in the interaction point The luminosity has to be concentrated inside the vertex detector in the vicinity of the interaction point. The collider has to be operated with a bunched beam at the r.m.s. bunch length $\sigma_{s}$ not longer than $60 \mathrm{~cm}$ to allow the vortex detector geometry. Only this case will be discussed below.

From the upper side, the peak luminosity is limited by detector electronics to avoid its overload by the useful signal from the interaction point. Thus, the maximum achievable event count rate $\dot{N}_{\text {event }}$ is limited to the level of about $7 \mathrm{kHz}$. In the NICA energy region, the nuclear reaction cross-section $\sigma$ is about $7 \cdot 10^{-24} \mathrm{~cm}^{-2}$. By definition, the corresponding luminosity $L_{D}$ is given by

$$
L_{D}=\dot{N}_{\text {event }} / \sigma
$$

that for our parameters corresponds to $L_{D} \leq 1 \cdot 10^{27} \mathrm{~cm}^{-2} \mathrm{~s}^{-1}$. Such a level is typical for other heavy-ion colliders: RHIC for Au-Au collisions at maximum energy and $\mathrm{LHC}$ for $\mathrm{Pb}-\mathrm{Pb}$ collisions. However, in NICA, the ion kinetic energy below $4.5 \mathrm{GeV} / \mathrm{u}$ is sufficiently smaller, leading to specific limitations for the collider parameters.

The collider working cycle has to be designed to provide a mean luminosity as close as possible to the peak one. The upper technical limit of the mean luminosity is determined by the ion production rate $\dot{N}_{\text {pr }}$ provided by the injection chain. During operation, all produced ions are lost in the accelerator or detector and for the loss rate we have $\dot{N}_{\text {loss }}=\dot{N}_{\text {pr }}$. Some part of the losses is related to the investigating reactions, another part - to different parasitic processes: losses at injection into the collider, scattering with residual gas atoms, and so on, thus $\dot{N}_{\text {loss }}=\dot{N}_{\text {event }}+\dot{N}_{\text {other loss. }}$ As a result, the mean luminosity cannot exceed the following value

$$
L \leq \dot{N}_{\mathrm{pr}} / \sigma .
$$

This limit can be of great importance for experiments with exotic particles: antiprotons or radioactive ions. NICA collider heavy ion injection chain is designed to provide $10^{9}$ Au nuclei each $5 \mathrm{sec}$. That corresponds to the limitation of the mean luminosity at the level of about $3 \cdot 10^{31} \mathrm{~cm}^{-2} \mathrm{~s}^{-1}$. Four orders of magnitude of the technical reserve permit the use of the NICA injection chain for a few experiments in parallel that are presumed by the experimental program.

At a large ion production rate, the collider mean and peak luminosity are limited by particle dynamics. For identical round beams, the peak luminosity is given by the following well-known formula:

$$
L=\frac{n_{b} N_{b}^{2}}{4 \pi \varepsilon \beta^{*} T_{\mathrm{rev}}} f\left(\frac{\sigma_{s}}{\beta^{*}}\right),
$$

where $T_{\text {rev }}$ is the ion revolution period in the collider, $f\left(\frac{\sigma_{s}}{\beta^{*}}\right)=\frac{1}{\sqrt{\pi}} \int_{-\infty}^{\infty} \frac{\exp \left(-u^{2}\right) d u}{\left[1+\left(\frac{u \sigma_{s}}{\beta^{*}}\right)^{2}\right]}$ is so-called the "hour-glass" factor related to the finite bunch length, all other parameters are discussed below. $n_{b}$ 
is the number of bunches circulating in each collider ring. To reach maximum luminosity, it has to be chosen as large as possible. Still, the inter-bunch distance is to be large enough to avoid parasitic collisions inside and outside the detector. The beta function in the collision point $\beta^{*}$ has to be as small as possible but comparable with bunch length $\sigma_{s}$ to avoid luminosity reduction due to the "hour-glass" factor.

At high energies of the colliding beams (Tevatron, RHIC, LHC), the collider is also used for the beam acceleration. In this case, a train of bunches (or a few trains) injecting into the collider at minimum energy is prepared by the injection chain. That leads to an obvious recommendation for the beam parameters to reach maximum luminosity:

- the bunch intensity $N_{b}$ has to be as large as possible,

- the growth of the beam emittance $\varepsilon$ has to be minimized in all elements of the injection chain.

Both these recommendations can be combined: to increase the luminosity, one has to increase the bunch brightness $N_{b} / \varepsilon$ at the exit of the injection chain.

\subsection{Space charge effects at low energy}

The bunch brightness is limited in the collider by two main space charge effects.

Repulsion between ions with the same sign of the electric charge leads to an incoherent shift of the betatron oscillation frequency. Change of the betatron oscillation number $Q$ is given by Laslett tune shift formula:

$$
\Delta Q=-\frac{Z^{2} r_{p}}{A} \frac{N_{b}}{4 \pi \varepsilon \beta^{2} \gamma^{3}} F_{\mathrm{sc}} F_{b},
$$

where $A, Z-$ the ion atomic and charge numbers, $r_{p}$ is the proton classical radius, $\beta, \gamma-$ relativistic parameters, $F_{\mathrm{sc}}$ is the image force correction factor depending on the vacuum chamber design and for estimations can be set to unity, $F_{b}=\frac{C}{\sqrt{2 \pi} \sigma_{s}}-$ bunching factor, $C$ is the ring circumference.

The second space charge effect is usually characterized by the so-called beam-beam parameter (linear part of the tune shift due to fields of the opposite bunch in the collision point) calculated in accordance with the following formula

$$
\xi=\frac{Z^{2} r_{p}}{A} \frac{N_{b}}{4 \pi \varepsilon \beta^{2} \gamma} \frac{1+\beta^{2}}{2} .
$$

Acceptable displacement of the ring working point (not leading to the particle loss or fast emittance growth) due to both these effects determines maximum achievable bunch brightness. If the injection chain cannot provide the limit value, the bunch brightness can be increased by beam cooling application in the collider, for instance, stochastic cooling implemented at RHIC.

The Lasslett tune shift decreases fast with an increase of the beam energy (as $\gamma^{3}$, see (3.2)), because the magnetic field of the beam compensates electrical repulsion between the ions. The beam-beam parameter decreases with energy as $\gamma$ (see (3.3)), because the magnetic field of the opposite bunch leads to an increase of the repulsion. As a result at high energy (RHIC, LHC) the Lasslett tune shift is negligible and the luminosity is limited by the beam-beam parameter. At low energy, the beam-beam parameter and Laslett tune shift can be comparable (typical for 
RHIC operation during the Beam Energy Scan program) or Laslett tune shift dominates (for NICA parameters, the beam-beam parameter is about one order of magnitude less than the Laslett tune shift). There is another important difference between these two effects: $\xi$ does not depend on the ring circumference $C$ and is determined by the number of the collision points, while the Laslett tune shift is linearly proportional to $C$ (via bunching factor in (3.2)).

At low energy, when the Laslett tune shift dominates, the beam brightness can be expressed from the maximum achievable tune shift $\Delta Q$ that gives for the luminosity:

$$
L=\frac{A}{Z^{2} r_{p}} \frac{N_{b} c}{\beta^{*}} \frac{\sqrt{2 \pi} \sigma_{s}}{C l_{b b}} \gamma^{3} \beta^{3} f\left(\frac{\sigma_{s}}{\beta^{*}}\right) \Delta Q
$$

Here $l_{b b}=\frac{C}{n_{b}}$ is the mean inter-bunch distance.

This formula permits the calculation of the maximum achievable peak luminosity when the maximum bunch intensity is constant and determined by injection chain performance. To reach this maximum value, the beam emittance must be varied with energy as follows:

$$
\varepsilon=\frac{Z^{2} r_{p}}{A} \frac{N_{b}}{4 \pi \beta^{2} \gamma^{3} \Delta Q} \frac{C}{\sqrt{2 \pi} \sigma_{s}}
$$

that can be achieved at the active formation of the beam phase volume only that can be provided by a beam cooling application. Such a regime of the collider operation, when the luminosity is limited by the bunch intensity, has an obvious technical limit at low energies. The emittance increases at energy decrease. When it begins to be equal to a maximum acceptable value determined by the collider acceptance, one needs to decrease the bunch intensity at further energy decrease.

A possibility to increase the bunch intensity with energy increase can give an additional increase of the luminosity. In this case, one can exclude the bunch intensity from the (3.4) and obtain:

$$
L=\left(\frac{A}{Z^{2} r_{p}}\right)^{2} \frac{\varepsilon}{\beta^{*}} \frac{8 \pi^{2} \sigma_{s}^{2} c}{C^{2} l_{b b}} \gamma^{6} \beta^{5} f\left(\frac{\sigma_{s}}{\beta^{*}}\right) \Delta Q^{2} .
$$

In this regime of operation, when the luminosity is limited by the acceptance, the bunch intensity should be varied with energy variation in accordance with the following formula:

$$
N_{b}=\frac{A}{Z^{2} r_{p}} \frac{\sqrt{2 \pi} \sigma_{s}}{C} 4 \pi \gamma^{3} \beta^{2} \varepsilon \Delta Q .
$$

Maximum usage of the available phase volume leads to the maximum achievable luminosity; however, this regime has physical limitation related to a coherent instability of the beam heaving large peak current.

Keeping the most important parameters in (3.6), one can conclude that in such a regime, the maximum peak luminosity is proportional to

$$
L \sim \frac{\varepsilon \Delta Q^{2}}{C^{2}} .
$$

In difference to a high energy collider (when the beam-beam parameter is dominated), to reach maximum peak luminosity, one needs to follow the next: 
- the ring circumference has to be as small as possible,

- the beam emittance has to be as large as possible (close to an acceptance limit),

- the bunch population should be varied with energy to provide the required bunch brightness,

- a beam cooling is mandatory during the bunch formation and during the experiment.

The usual requirement to have a working point far from low order resonances becomes to be stronger because of the quadratic dependence of the luminosity on the achievable tune shift. For instance, for the NICA collider both tunes were chosen below half integer resonance and the achievable tune shift (which is calculated as a sum of the Laslett tune shift and two beam-beam parameters corresponding to two collision points) was estimated as 0.05 .

\subsection{Regimes of the collider operation}

Both formulae (4 and 6) describing so-called "space-charge dominated regime (SC DR)" show a fast increase of the luminosity with the increase of the beam kinetic energy. At some energy, the luminosity reaches the value $L_{D}$ corresponding to optimum detector operation.

At the energy larger than this value, it is not necessary to have the bunch parameters corresponding to the maximum achievable tune shift value. Having flexibility in setting the tune-shift, one can re-optimize the bunch to minimize processes leading to the luminosity dilution during the experiment in order to have a mean luminosity close to peak value. All the processes leading to the increase of the bunch phase space and decreasing the bunch intensity are well known. But in difference to a high energy collider, the most important process is Intra-Beam Scattering (IBS).

At the LHC, the IBS is negligible practically. At RHIC (during operation at maximum energy without stochastic cooling), the characteristic time of the momentum spread growth due to IBS was about 4 hours. At NICA energy range, the expected growth time lies in the range from a few tens of seconds up to about $30 \mathrm{~min}$. Therefore the collider operation at energy larger than the SC DR border is called IBS dominated regime (IBS DR). To suppress the IBS process a beam cooling during the experiment is mandatory.

To maximize the growth time and make requirements to a cooling system weaker, the bunch parameters have to be provided near thermo-dynamical equilibrium between longitudinal and transverse degrees of freedom (to avoid fast relaxation). It means that the ratio between the bunch momentum spread (determining the temperature of the longitudinal degree of freedom) and transverse emittance (a measure of the temperature of the transverse degree of freedom) has to be equal to same exact value depending on the beam energy and strongly influenced by the ring lattice structure.

During the collider optic design, significant efforts should be made for the IBS optimization, and all the modifications provided during development have to be proved from the side of their influence on the equilibrium bunch parameters and the IBS growth times.

The energy corresponding to the border between SC DR and IBS DR is one of the most important parameters of the collider operated at low energy. The border is determined by the accelerator and detector parameters together. For instance, at the STAR experiment (maximum count rate is about $1 \mathrm{kHz}$ ) during the RHIC energy scan program, this border corresponds to the gold nuclei kinetic energy of about $10 \mathrm{GeV} / \mathrm{u}$ and dominated mainly by the ring circumference. 


\section{Two concepts of the low energy collider}

The low-energy collider can be designed based on one of two alternative concepts. The first is to provide as small as possible ring circumference using large field superconducting magnets and reach maximum possible luminosity independently of the detector requirements. The second one - is to consider the maximum detector count rate and choose the collider parameters corresponding to the required border between SC DR and IBS DR.

\subsection{NICA CDR}

The first concept was developed in the NICA conceptual design report and presented in [4]. The simplest choice for the collider geometry with two crossing points is two identical rings separated vertically, having a racetrack structure: two arcs and two long straight sections. Minimum arc diameter corresponds to the large magnetic field of the bending magnets. Therefore the first NICA concept was based on curved twin-bore magnets of $\cos \theta$ - style at the maximum field of 4-5 T (figure 2, right). This level of the field permits to locate the ring of $200-250 \mathrm{~m}$ of circumference in the existing JINR experimental building (figure 2, left).
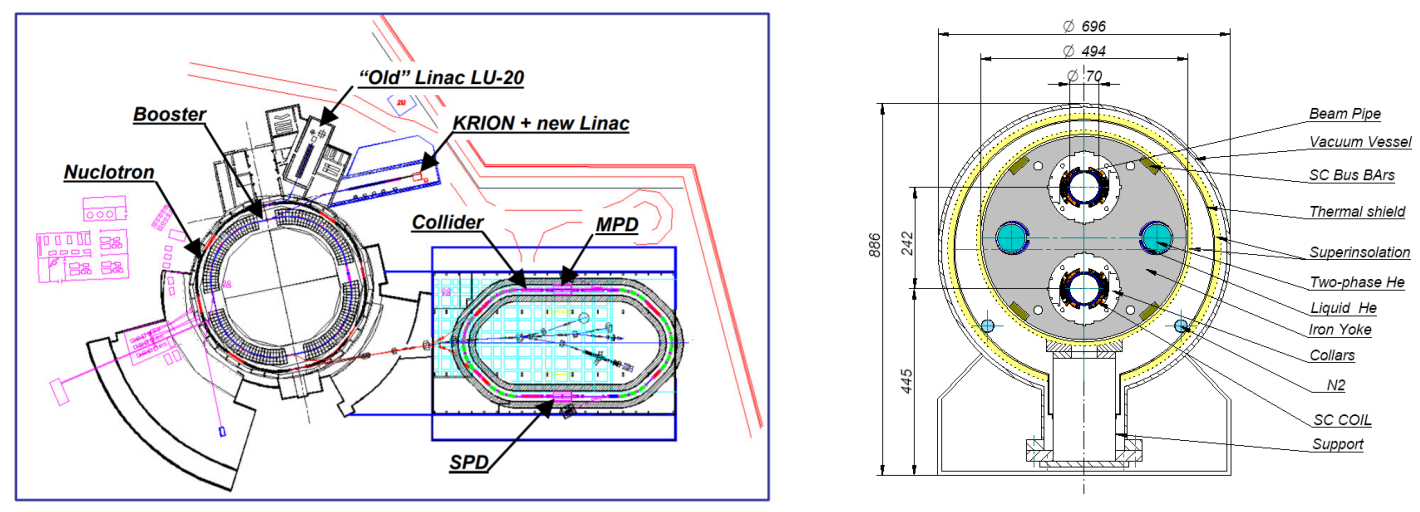

Figure 2. Location of the NICA elements in the existing buildings (left) and a preliminary design of the NICA collider twin-bore $5 \mathrm{~T}$ dipole (right).

In this concept, each element of the facility provided its own function.

The Booster provided required bunch intensity and phase volume. The Booster acceptance permitted storage of the beam at injection, and a conventional electron cooling system is sufficient to decrease the bunch longitudinal emittance to the value required in the collider.

The Nuclotron was used for the bunch acceleration and the bunch compression before transfer to the collider.

The collider provided stabilization of the bunch parameters during a long circulation period. An electron cooling system and one RF cavity per ring are necessary for this. Storage of the required bunch number is performed using the bunch-to-bucket injection scheme.

The efficiency of the cooling application was estimated at $3.5 \mathrm{GeV} / \mathrm{u}$ for the bunch parameters expected at the exit of the injection chain: the bunch intensity is up to $10^{9}$ ions, emittance is about $0.3 \pi \cdot \mathrm{mm} \cdot \mathrm{mrad}$, the bunch length is $30 \mathrm{~cm}$. The optic structure was not optimized for minimum IBS growth rates. The bunch parameters are far from the thermal equilibrium. Calculated heating times 
are about $10-50 \mathrm{~s}$. At such parameters a stochastic cooling application is unpracticed. At an electron cooling application to suppress the ion-electron recombination the transverse electron temperature has to be about $50 \mathrm{eV}$. Therefore the required cooling rate can be provided using magnetized cooling only. Simulations showed that at the field in the cooling section of $2 \mathrm{~T}$ and the electron current of $0.5 \mathrm{~A}$, the operation at stable luminosity can be provided for about 1 hour (figure 3 ).

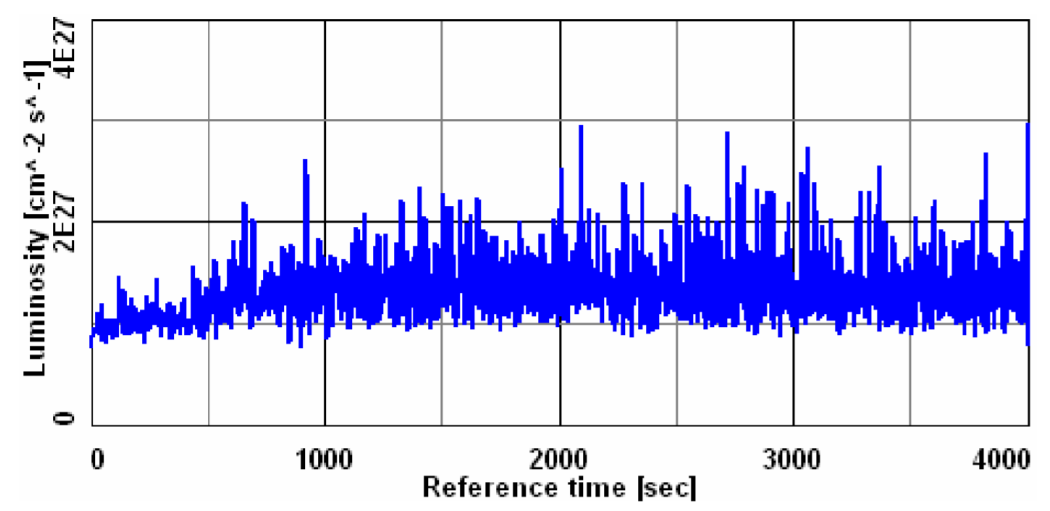

Figure 3. BETACOOL simulation of the luminosity time dependence at common action of IBS and electron cooling [5].

R\&D work for the large field magnets has been started [6]. However, the development of technology requires a long period of time. Therefore the NICA collider technical design was developed based on an alternative concept.

\subsection{Present concept}

The most important kinetic energy for MPD experiment is about $3.5 \mathrm{GeV} / \mathrm{u}$. Correspondingly the collider can be designed to provide the border between SC DR and IBS DR at an energy below this value. It can be done at the ring circumference of about $500 \mathrm{~m}$, based on super-ferric magnets at $1.8 \mathrm{~T}$ of the maximum field. Initially, it was shown by FNAL experts, and the concept was developed in cooperation with FNAL and FZJ [7].

To have the border between SC DR and IBS DR at the energy of about $3 \mathrm{GeV} / \mathrm{u}$, the bunch intensity has to be increased up to $2 \div 3 \cdot 10^{9}$ ions, which is larger than the bunch intensity expected from the collider injection chain. A possible solution to this problem is to provide the beam stacking inside the collider and then provide the beam bunching and formation of short bunches required for the collisions. In this case, instead of one cavity per ring in the NICA CDR version, the beam storage and bunch formation requires one barrier bucket cavity, 4 cavities working at harmonics equal to the bunch number, and 8 cavities operated during collisions [8]. The long straight section of the collider at large circumference permits to locate the required equipment.

A collider lattice development overcame several iterations, which took into account an interdependency of major machine parameters. The IBS is one of the major phenomena limiting the luminosity lifetime. It determines the requirements of the beam cooling systems and directly influences the machine operation scenario. The minimum of the IBS heating rates corresponds to the quasi-equilibrium point for an optics structure defined by the equality of averaged IBS times $\tau_{\mathrm{IBSx}}=\tau_{\mathrm{IBSy}}=\tau_{\mathrm{IBSp}}$. The IBS rates were investigated for a few versions of FODO and ODFDO- 
(triplet) lattices. Basing on the results of the studies, the FODO structure with 24 cells was chosen as the basis for NICA collider optics. The IBS growth time in the IBS DR was increased more than one order of magnitude compared to the CDR version and reached 600-2000 s (figure 4). As a result, the beam cooling can be effectively provided by both methods: electron or stochastic.

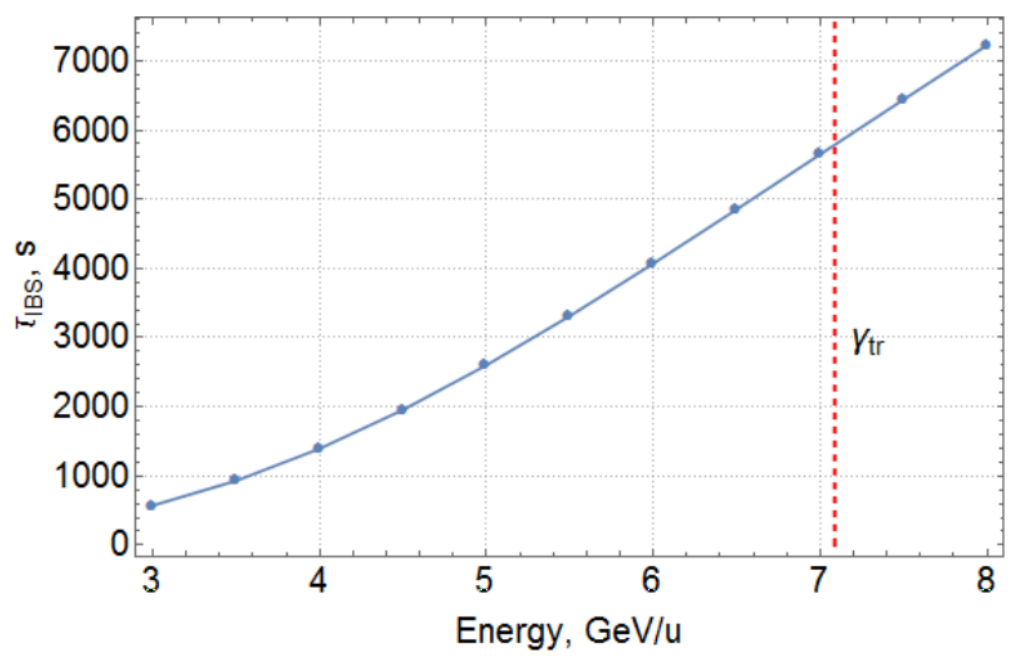

Figure 4. IBS time versus energy for NICA collider lattice at quasi-equilibrium. The beam intensity is $2 \cdot 10^{9}$ particles per bunch [9].

The requirement to have the beam emittance as large as possible leads to careful optimization of the lattice structure in order to obtain large dynamic aperture (DA). Aperture of the NICA collider vacuum chamber was chosen to have a geometrical acceptance equal to $40 \pi \cdot \mathrm{mm} \cdot \mathrm{mrad}$. The maximum acceptable emittance was estimated as $1.1 \pi \cdot \mathrm{mm} \cdot \mathrm{mrad}$ that corresponds to the r.m.s. bunch size 6 times less than the aperture. The NICA collider DA was investigated taking into account all possible nonlinearities of the magnetic field. The fringe fields of the magnetic elements, particularly the final focus quadrupoles, showed the most severe effect [10]. The effect is very sensitive to the beta function in the collision point $\beta^{*}$, so at the $\beta^{*}=0.35 \mathrm{~m}$ the stable DA is less than geometrical acceptance of the ring, when the optics with the $\beta^{*}=0.60 \mathrm{~m}$ provides the double excess of DA over the geometrical acceptance. Due to peculiarities of the collider operation in the IBS DR, at the change of the $\beta^{*}$, the luminosity can be kept at the same level by a corresponding increase of the bunch intensity. Variation of the $\beta^{*}$ leads to shifting of the border between SC DR and IBS DR. For the NICA parameters, the change of $\beta^{*}$ from 0.35 to $0.60 \mathrm{~m}$ corresponds to shift from $3 \mathrm{GeV} / \mathrm{u}$ to about $3.2 \mathrm{GeV} / \mathrm{u}$.

The last version of the collider structure with results of simulations is presented in the article [11].

\section{Outlook}

Construction of the NICA is the flagship project for JINR at present. To date, the modernization of the Nuclotron light ion injection chain is completed. A new linear accelerator for the heavy ion injection chain was constructed and commissioned in 2016. First experiments at BM@N detector 
and radiobiology experiments in the frame of the NICA research program were performed in Spring of 2018 with carbon, argon, and krypton beams. All superconducting magnets for the Booster were fabricated at JINR and the Booster ring was commissioned with the beam in 2020. The first run of the Nuclotron with the injection of the high-intensity heavy-ion beam from the Booster is scheduled for 2021. Construction of the NICA collider started in 2016, it is progressing well and is on track for completion in 2023.

\section{Acknowledgments}

Authors express their gratitude to V. Lebedev, S. Nagaitsev, and Yu. Senichev for the help at all stages of the NICA accelerator complex design, and to P. Ostroumov and V. Shiltsev for careful reading the manuscript and useful comments.

\section{References}

[1] http://nica.jinr.ru/.

[2] A. Sorin, V. Kekelidze, A. Kovalenko, R. Lednicky, I. Meshkov and G. Trubnikov, Heavy-ion program at NICA/MPD at JINR, Nucl. Phys. A 855 (2011) 510.

[3] https://edu.jinr.ru/.

[4] G. Trubnikov et al., Project of the nuclotron-based ion collider facility (NICA) at JINR, in proceedings of EPACO8, Genoa, Italy, 23-27 June 2008, pp. 2581-2583.

[5] E. Ahmanova et al., Application of cooling methods to NICA project, in proceedings of COOLO9, Lanzhou, China, 31 August - 4 September 2009, pp. 16-20.

[6] NICA collaboration, Design of a twin-aperture 4 T curved dipole based on high current hollow superconducting cables for the NICA collider at JINR, J. Phys. Conf. Ser. 234 (2010) 032033.

[7] S. Kostromin et al., Lattice of NICA collider rings, in proceedings of IPAC'10, Kyoto, Japan, 23-28 May 2010, pp. 690-692.

[8] A.V. Eliseev et al., Storage, acceleration and short bunched beam formation of ${ }_{197} A u^{+79}$ ions in the NICA collider, in proceedings of RUPAC2012, Saint-Petersburg, Russia, 24-28 September 2012, pp. 30-32.

[9] S. Kostromin et al., IBS near transition energy, in proceedings of HB2018, Daejeon, Republic of Korea, 17-22 June 2018, pp. 252-255.

[10] O. Kozlov et al., Correction of the magnetic field in the NICA collider, in proceedings of RUPAC2018, Protvino, Russia, 1-5 October 2018, pp. 149-151.

[11] S.A. Kostromin et al., Optimization the optical structure of the NICA collider, Phys. Part. Nucl. Lett. 17 (2020) 447. 